\title{
Retinal Microvascular Structure and Function in
}

\section{Patients with Risk Factors of Atherosclerosis and Coronary Artery}

\section{Disease}

Ali H Al-Fiadh ${ }^{1,2}$, Omar Farouque ${ }^{1,2}$, Ryo Kawasaki ${ }^{3,4}$, TT Nguyen ${ }^{3}$, Nazim Uddin ${ }^{1}$, Melanie Freeman ${ }^{2}$, Sheila K. Patel ${ }^{2}$, Louise M Burrell $^{1,2}$, Tien Y. Wong ${ }^{3,5}$

${ }^{1}$ Department of Cardiology, Austin Health, Heidelberg VIC 3084, Australia;

${ }^{2}$ Department of Medicine, University of Melbourne, Australia; ${ }^{3}$ Centre for Eye Research Australia, East Melbourne VIC 3002, Australia; ${ }^{4}$ Department of Public Health, Yamagata University, Japan; ${ }^{5}$ Singapore Eye Research Institute, National University of Singapore, Singapore

Tables in manuscript $=2$; Figures in manuscript $=4$

Corresponding author: A/Prof Omar Farouque Department of Cardiology, Austin Health, 145 Studley Road, Heidelberg VIC 3084, Australia

Email: omar.farouque@austin.org.au

Tel: +61-3-9496-3034

Fax: +61-3-9457-0934 


\section{Abstract}

Objective: Retinal microvascular signs are markers of cardiovascular disease risk. There are limited data, on relationships between retinal microvascular signs and retinal microvascular endothelial function. We sought to determine the relationship of retinal vascular signs with retinal microvascular endothelial function in patients with or at high risk of coronary artery disease. Methods: Participants with atherosclerosis risk factors and coronary disease $(n=258$; mean age $57 \pm 11$ years $)$ were recruited to have static and dynamic retinal vascular assessment. Retinal arteriolar dilatation in response to flicker light (FI-RAD) was measured using the Digital Vessel Analyzer and expressed as percentage increase over baseline diameter. Static retinal photographs were acquired utilising a digital fundus camera for measurement of central retinal artery and vein equivalent (CRAE and CRVE), arteriovenous nicking (AVN) and focal arteriolar narrowing (FAN). Results: Intra-class correlation coefficient was 0.82 for flicker-light induced retinal arteriolar dilatation. There were modest associations in retinal vascular measurements between eyes. For each $10 \mu \mathrm{m}$ decrease in retinal arteriolar diameter, the absolute increase in FI-RAD was $0.28 \%$ $(95 \%$ CI $0.11,0.45 ; p=0.002)$ independent of age, gender and atherosclerosis risk factors. AVN and FAN were associated with attenuated FI-RAD $(\beta=-0.67 \% ; 95 \% \mathrm{CI}$ $-1.20,-0.15 ; p=0.012)$ and $(\beta=-0.83 \% ; 95 \%$ CI- $1.44,-0.23 ; p=0.007)$ respectively after adjustment for age and gender. Conclusion: Assessment of retinal microvascular endothelial function is reproducible and correlated with retinal microvascular structure and signs, independent of atherosclerosis risk factors. Assessment of retinal vascular structure and function may provide insights into atherosclerotic disease.

Key words: retinal vessels, microcirculation, endothelial function, coronary disease 


\section{Introduction}

The retinal circulation is unique as it allows a direct and non-invasive window to the health of the human circulation in vivo. Large epidemiologic studies have shown that retinal microvascular signs (e.g., arteriovenous nicking, focal arteriolar narrowing, and measurement of static retinal vascular calibre) provides information on risk of vascular complications, cardiac events and stroke in the general population ${ }^{1-6}$.

The mechanisms and pathways underlying these associations remain, however, unclear. Advances in retinal vascular imaging technology have allowed retinal microvascular function, a nitric oxide dependent phenomenon, to be evaluated in "real time" by non-invasive dynamic assessment of retinal vascular dilatation in response to diffuse luminance flicker light using the Dynamic Vessel Analyzer (DVA) ${ }^{7}$. There are limited data on how static retinal microvascular signs are correlated with retinal microvascular endothelial function, particularly in subjects with or at risk for coronary heart disease ${ }^{8}$. Understanding the associations between static and dynamic retinal vascular markers may help in further developing a diagnostic tool capable of early detection of atherosclerosis, and assist in risk stratification of patients with cardiovascular disease.

To address these gaps, we sought to examine the feasibility of measuring retinal microvascular endothelial function using the DVA in patients with or at risk of coronary artery disease, and to study the inter-relationship between retinal vascular structure and function. 


\section{Materials and methods}

\subsection{Study population and patient preparation}

The study was approved by the Human Research Ethics Committee of Austin Health and written informed consent was obtained from all subjects. All participants $(\mathrm{n}=$ 258) underwent retinal photography, including static and dynamic assessment. The inclusion criteria for recruitment were that participants had to have at least two traditional cardiovascular risk factors and/or clinically evident coronary artery disease. Traditional cardiovascular risk factors included a history of hypertension, dyslipidaemia, diabetes mellitus, active cigarette smoking history or a family history of premature coronary disease. Clinical evident coronary disease was defined as either a) coronary artery stenosis of $>50 \%$ documented at coronary angiography, b) the presence of symptoms of myocardial ischaemia and an abnormal stress test, or c) an acute coronary syndrome. Patients were excluded if an adequate retinal examination could not be performed due to cataracts, narrow-angle glaucoma or history of epilepsy. All tests were undertaken in the morning after an overnight fast, in a quiet, temperature-controlled environment. Vasoactive drugs, caffeinated drinks and nicotine were withheld when possible for at least 12 hours, to minimise the potential influence of these factors on vessel calibre.

\subsection{Dynamic and static retinal vascular imaging}

Retinal photographs and dynamic retinal vascular assessment were performed on both eyes in all subjects after the pupils were dilated with $1 \%$ tropicamide eye drop solution in order to obtain optimal retinal images. Assessment of flicker-light induced retinal vasodilatation was performed using the DVA (IMEDOS, Germany) to assess percentage change of retinal vessel diameter compared to baseline ${ }^{9}$. In brief, the 
patient focuses at a fixation bar positioned inside its viewing system while the fundus is examined under green light. An arteriolar and venular segment between half and two disc diameters from the margin of the optic disc is selected. The measurement starts automatically at a video frequency of $25 \mathrm{~Hz}$ which allows 25 readings of vessel diameter per second. The diameter of the vessel was then calculated continuously along the selected segment, which is preferably $1.5 \mathrm{~mm}$ in length. A baseline recording for 50 seconds was performed followed by a 20 second provocation by flickering light of the same wavelength at a frequency of $12.5 \mathrm{~Hz}$. This was then followed by 80 seconds of steady illumination to allow the vessel to return to baseline. The cycle is then repeated twice with a total duration of 350 seconds (Figure 1). Responses were measured in each eye and recorded for comparison. A complete examination was performed in approximately 20 minutes.

Static digital retinal photographs were taken of both eyes after pupil dilation (Canon EOS 40D, CF-60UVi fundus camera). A computer-based standardized protocol (IVAN, University of Wisconsin, USA) automatically measures the diameter of arterioles and venules within $1 / 2$ to 1 disc diameter from the margin of the optic disc to summarize arteriolar and venular diameter ${ }^{10}$. The central retinal artery and vein equivalent (CRAE and CRVE) is calculated from an average of the biggest six vessels reflecting the estimated diameter of the central retinal artery and vein ${ }^{11}$. Other structural changes were also assessed, including focal arteriolar narrowing (FAN) and arteriovenous nicking (AVN) following the modified protocol from the Multi-Ethnic Study of Atherosclerosis ${ }^{12}$. 


\subsection{Reproducibility of dynamic retinal studies}

In a subset of 13 patients ( $38 \%$ male; mean age of $51 \pm 16$ years) with atherosclerosis risk factors, dynamic retinal studies were performed by the same observer and repeated after 6 weeks while medication was held constant. Intraobserver intraclass correlation coefficient (ICC) analysis was performed to assess test-retest reproducibility. ICC of 0-0.2 indicates poor agreement, 0.3-0.4 indicates fair agreement, 0.5-0.6 indicates moderate agreement, 0.7-0.8 indicates strong agreement, and $>0.8$ indicates almost perfect agreement ${ }^{13}$. Furthermore, agreement between $1^{\text {st }}$ and $2^{\text {nd }}$ measurements for baseline diameter and maximum dilatation of retinal arterioles and venules in the same 13 subjects was assessed, using Bland-Altman analysis ${ }^{14}$. A test is considered reproducible if the mean difference is close to zero and $95 \%$ of differences are less than two standard deviations.

\subsection{Statistical analysis}

Continuous variables were expressed as mean \pm SD and categorical variables were expressed as count (\%). Data were assessed for normality using the Shapiro-Wilk test and found to be normally distributed. Dynamic and static retinal vascular variables were assessed in both eyes. The relationship between the right and left eye was evaluated first and average measurements were used when appropriate to assess relationships between variables as described below. Cohen's Kappa coefficient was calculated as a statistical measure of agreement between categorical data. Pearson's correlation coefficient was used to assess associations between continuous variables. Data for FAN and AVN were dichotomized into present versus absent. Linear regression analysis was performed to assess the relationship between FI-RAD, CRAE, 
CRVE, FAN and AVN in each eye adjusting for cardiovascular risk factors, which were selected based on their known association with vascular function. Each outcome variable was entered separately into the multivariate analysis adjusting for age, gender, systolic blood pressure, BMI, smoking, family history of ischaemic heart disease, hypertension, dyslipidaemia, serum glucose, creatinine and cholesterol levels, statin therapy, renin-angiotensin blocker, beta-blocker and antiplatelet therapy. A $p$ value of $\leq 0.05$ was considered statistically significant. All statistical analyses were performed using SPSS version 18 for Windows (SPSS Inc., Chicago, IL, USA).

\section{Results}

\subsection{Reproducibility and association between eyes}

The characteristics of the study population are displayed in Table 1. ICC was 0.99 and 0.98 for baseline arteriolar and venular diameter respectively, and 0.82 and 0.79 for flicker-light induced retinal arteriolar and venular dilatation respectively. None of 13 baseline retinal arteriolar and venular diameter measurements were beyond the limits of agreement while at maximum dilatation, 1/13 of the arteriolar (FI-RAD), but not venular (FI-RVD), measurements were beyond the limits of agreement (Figure 2). Reproducibility was similar in the left and right eyes.

The association in static and dynamic vascular measurements between the right and left eyes are displayed in Table 2 . There is a modest and statistically significant association between parameters from right and left eyes $(p<0.001)$. Figure 3A demonstrates the relationship between FI-RAD in the left and right eyes. Differences between eyes were similar in patients with established coronary disease or risk factors alone with correlation coefficients of 0.548 and 0.572 respectively. 


\subsection{Relationship between static and dynamic retinal parameters}

Smaller BL-RAD was associated with larger FI-RAD (Figure 3B). In the overall study population, for each $10 \mu \mathrm{m}$ decrease in BL-RAD, the absolute increase in FIRAD was $0.28 \%$ (95\% CI $0.11,0.45 ; p=0.002)$ independent of age, gender, mean blood pressure, pulse pressure, BMI, smoking, dyslipidaemia, serum glucose, cholesterol level and medication use.

In addition to baseline diameter, age and serum glucose levels were associated with attenuated FI-RAD response. For each 10 year increment in age, FI-RAD decreased by an absolute $0.66 \%(95 \% \mathrm{CI}-0.91,-0.41 ; p<0.001)$ and for each $1 \mathrm{mmol} / \mathrm{L}$ increase in serum glucose, FI-RAD decreased by an absolute $0.17 \%$ (95 CI -0.26, -0.07; $p=$ $0.001)$.

When stratified according to gender, the association between BL-RAD and FI-RAD was stronger in females than males $(r=-0.26 ; p=0.020$ vs $r=-0.12 ; p=0.106)$. Stratification by statin therapy was also assessed and the association between BLRAD and FI-RAD was found in patients on statin therapy compared to those who were not on statin therapy ( $r=-0.28 ; p=0.001$ vs $r=0.01 ; p=0.984$ respectively).

When stratified according to age ( $\leq 60$ years or $>60$ years), the association between BL-RAD and FI-RAD was similar $(r=-0.17 ; p=0.067$ s $r=-0.17 ; p=0.044)$.

Smaller BL-RVD was associated with larger FI-RVD. For each $10 \mu \mathrm{m}$ decrease in BLRVD, the absolute increase in FI-RVD was $0.15 \%$ (95\% CI 0.01, 0.28; $p=0.037$ ). When stratified according to gender and age, the association between BL-RVD and FI-RVD was no longer statistically significant in either group. When stratified by statin therapy, it was found that the association between BL-RVD and FI-RVD was 
stronger among those who were on statin therapy compared to statin naïve patients $(r$ $=-0.23 ; p=0.004$ vs $r=0.08 ; p=0.411)$. There was a weak but statistically significant positive association between FI-RAD and FI-RVD $(r=0.27 ; p<0.001)$.

In patients with AVN, there was an absolute reduction in FI-RAD of $0.86 \%$ (95\% CI $1.40,-0.31 ; p=0.002$ ) compared to those with no AVN in the left eye (Figure 4). This relationship continued to be significant after adjustment for age and gender $(\beta=-$ $0.67 \% ; 95 \% \mathrm{CI}-1.20,-0.15 ; p=0.012)$. There was a trend to absolute reduction in FIRAD of $0.50 \%(95 \%$ CI-1.05, 0.04; $p=0.069)$ in subjects with AVN in the right eye. Patients with FAN had an absolute reduction in FI-RAD of $0.83 \%$ (95\% CI-1.44, $0.23 ; p=0.007)$ after adjustment for age and gender in the left eye (Figure 4).

Similarly, there was a trend to absolute reduction in FI-RAD of $0.60 \%$ in subjects with FAN in the right eye (95\% CI-1.23, $0.04 ; p=0.065)$.

\subsection{Relationship between dynamic parameters and severity of coronary disease}

Whether retinal vascular reactivity differs according to severity and extent of coronary disease is unknown. To examine this question in our study population, we analysed the group of patients with coronary disease who had coronary revascularisation procedures $(n=74)$. An analysis was undertaken making the assumption that patients undergoing $\mathrm{CABG}$ have more severe and extensive disease than those undergoing percutaneous coronary intervention. There was no difference between these groups. FI-RAD was $1.62 \pm 1.52 \%$ vs $1.60 \pm 1.49 \%(p=0.97)$ and FIRVD was $4.12 \pm 2.23 \%$ vs $4.29 \pm 1.31 \%(p=0.77)$ in the PCI and CABG groups respectively. 


\section{Discussion}

In this study of patients with or at high risk of coronary artery disease, we investigated the reproducibility of retinal microvascular endothelial function assessment using the DVA and studied the inter-relationship between retinal vascular function and structure. The main findings are as follows. First, we showed that baseline and flicker light induced retinal arteriolar and venular dilatation, utilising the DVA, is reproducible. Second, we found modest to strong statistically significant linear associations between retinal vascular measurements between right and left eyes. Third, we found that smaller baseline retinal arteriolar and venular diameters were associated with larger microvascular dilator responses to flicker light. Finally, microvascular retinal signs of AVN and FAN were associated with reduced flicker light retinal arteriolar dilatation.

Although flicker-light induced retinal vasodilatation as measured by DVA has previously been shown to be reproducible in healthy subjects ${ }^{9,15}$, to our knowledge there have been no published reproducibility data in high risk patients with atherosclerosis and coronary artery disease. In this study we have shown that the measurement of retinal vessel calibre at baseline and in response to flicker using the DVA retinal camera is reproducible in this complex group of patients. This finding extends the measurement validity of the DVA to patients with atherosclerosis risk factors, as that in healthy subjects without risk factors.

The association of retinal vascular measures between right and left eyes suggests that measurements from a single eye may be adequate although not optimal to describe retinal vascular structure and physiology. There were some differences noted, which may have been due to methodological factors. For example, static and dynamic 
measurements were acquired at slightly different time points with resulting physiological variations in blood pressure and neural activity influencing retinal vessel diameter ${ }^{16-18}$. Refractive errors and intraocular pressure may also have been different between eyes and this may add some variation to retinal vessel diameter and dilator response ${ }^{19}$. However, this would not be expected to affect the relationship between retinal vascular changes and atherosclerosis risk factors ${ }^{20}$. Grader variability may also introduce further variation ${ }^{21}$. As we have shown in our studies, the right eye was more likely to have AVN and FAN than the left eye and although this was not statistically significant, these differences might contribute to the variation of dynamic retinal vascular response to flicker light between eyes. Dissimilarity in retinal vascular changes may also be related to biological differences. Endothelial dysfunction may affect the vascular tree in a heterogeneous manner and may also be different within segments of the same vessel due to local vascular factors such as branching, tortuosity ${ }^{22}$, and local pathology resulting in disturbances in flow patterns and occurrence of shear stress.

Such regional variability in vasomotion within the same vessel has been described in the coronary circulation ${ }^{23}$. In other circulatory beds, variations in response have also been noted. For example, differences between right and left carotid IMT have been detected and are modest with a correlation coefficient of $0.25-0.43$, which is lower than in our study ${ }^{24}$. In addition, heterogeneity has been described for the phenomenon of FMD within and between conduit arteries, which may be related to differences in baseline arterial diameter ${ }^{25}$.

Baseline retinal vascular diameter measured by the DVA was smaller than retinal vessel calibre measured from retinal photographs. This is a consequence of the 
different methodology used for vessel calibre measurement between these techniques.

CRAE and CRVE were measured using computer-assisted analysis techniques, which summarizes the individual retinal vessels into central retinal artery and vein equivalent. In contrast, the DVA measurement reflects the diameter of the intravascular column of red blood cells under the assumption that they are homogenously distributed ${ }^{9}$. The red blood cells are separated from the wall by a plasma edge stream. Additionally, the retinal vessel selected for dynamic studies is located half to two disc diameter from the optic disc margin. Although, it is approximately comparable to the site of measurement for the retinal vessel calibre, CRAE and CRVE are an average of the largest six vessels rather than a single arteriole and venule selected for assessment by the DVA.

Smaller baseline retinal arteriolar and venular diameter was associated with larger flicker-light induced retinal arteriolar and venular dilatation, respectively. This finding was statistically significant and independent of atherosclerosis risk factors and cardiovascular medication use. The flicker-light technique is a provocation test that results in vasodilatation of retinal arterioles and venules by increasing retinal blood flow, and is a nitric oxide dependent phenomenon ${ }^{7}$. It is a measure of retinal microvascular endothelial function and shares some similarity in mechanism to flow mediated dilatation of conduit vessels such as the brachial artery. In the ultrasound based brachial artery FMD technique, baseline brachial artery diameter has been shown to inversely correlate with FMD percentage increase ${ }^{25}$. This relationship might be explained by the observation that smaller vessels are subject to a higher shear stress stimulus and subsequently greater vasodilation, which may be mediated by nitric oxide or other vasoactive factors such as prostacyclin or endothelium-derived hyperpolarizing factor ${ }^{26}$. Another explanation could be related to the mathematical 
calculation of the flicker-light retinal vessel response as baseline diameter is a variable in the denominator of the equation and accordingly, the smaller the baseline diameter the larger the flicker response.

The inverse association between baseline retinal arteriolar diameter and flicker light response varied according to gender and statin therapy. Subjects who were women and on statin therapy had a stronger inverse relationship between BL-RAD and FIRAD. Conversely, no relationship was found between BL-RAD and FI-RAD in men, and statin naïve patients. Large epidemiologic studies have shown that smaller calibre retinal arterioles are associated with greater risk of cardiovascular events. However, smaller baseline retinal arteriolar calibre is also associated with larger FI-RAD, which signifies better microvascular endothelial function and is thought to be a protective factor. Our findings provide a potential explanation for this apparent paradox, as among subjects with some clinical characteristics associated with a higher risk of future cardiovascular events (men, and statin naïve patients), smaller calibre vessels did not associate with FI-RAD.

Although our findings are consistent with those from patients with diabetes ${ }^{8}$, they are at variance with results reported by Polak et al and Nagel et al ${ }^{27,28}$. These studies contained a small number of participants, which might not have been sufficient to demonstrate a significant relationship. Polak et al also demonstrated an inverse association between baseline retinal venular diameter and FI-RVD when $8 \mathrm{~Hz}$ of flicker stimulation was used, however, there was no association found when $16 \mathrm{~Hz}$ frequency was used. Although this frequency is still within the range required to stimulate retinal vessel dilatation ${ }^{29}$, the currently recommended flicker frequency is $12.5 \mathrm{~Hz}$, and that may affect the degree of vascular response to flicker light. Nagel et 
al looked at six eyes and selected retinal arterioles smaller than $85 \mu \mathrm{m}$ to compare with larger retinal arterioles with a baseline mean diameter of $115 \mu \mathrm{m}$. The flicker response of the smaller arterioles was less than that seen in the larger arterioles.

An important observation in our study was the association between AVN and FAN with reduced retinal arteriolar dilatation to flicker light. This finding indicates that structural retinal microvascular signs and retinal vascular function are linked.

Previous work has shown that FAN and AVN are markers of microcirculatory dysfunction, inflammation and perhaps endothelial dysfunction ${ }^{30}$, which may explain the association with impaired retinal microvascular endothelial function observed in our study.

There was no difference in FI-RAD and FI-RVD according to severity of obstructive coronary disease when assessed using a surrogate measure of coronary disease severity. We have reported a similar lack of association between severity of coronary artery disease with static retinal vascular calibre in a previous study ${ }^{31}$.

Study limitations. For clinical reasons, vasoactive medications were not withheld in the group of participants with ACS $(n=61)$, which may have had an impact on retinal vasomotor function. However, baseline vessel diameter was not different compared to those with non-ACS suggesting a lack of effect of medication on resting retinal vascular tone and statistical adjustment for medication use was performed. Furthermore, previous work has shown that calcium channel blockade and angiotensin converting enzyme inhibition did not affect the retinal vascular responses to flicker light ${ }^{32}$, which may be an advantage of this technique over others that assess endothelial function. Although attenuated flicker light retinal arteriolar and venular dilatation is thought to be due to endothelial dysfunction, previous clinical studies 
indicate that blocking nitric oxide synthesis attenuated but did not completely abolish retinal arteriolar and venular dilatation in response to flicker light in the human retina $^{7}$. This suggests that other pathways of vasomotion, which may or may not be dependent on the endothelium, and perhaps alternative factors like neuronal degeneration might also be responsible ${ }^{33}$. An electroretinogram, which is a mass electrical response of the retina to photic stimulation, may be able to overcome this limitation and differentiate between retinal vascular and neuronal response mechanisms ${ }^{34}$.

An endothelial function test that is accurate and easy to perform would be of value to overcome some limitations that exist in current techniques as described in detail elsewhere ${ }^{35}$. Due to advances in retinal imaging and the unique ability to view realtime microvascular function in a non-invasive and reproducible manner, the retinal circulation is a prime candidate for this purpose. A large-scale prospective outcomes study to examine the proposition that non-invasive assessment of retinal microvascular function can predict future cardiovascular events is needed. If proven, dynamic retinal vascular examination may become an important clinical tool in the assessment and risk stratification of cardiovascular disease.

In conclusion, this study demonstrates that DVA measurements to assess retinal microvascular endothelial function are reproducible in patients at risk of and with coronary artery disease. Both right and left eyes respond in a similar manner. Smaller retinal vessels were associated with an increased flicker response, which might be related to an increased shear stress in smaller diameter vessels. Finally, retinal microvascular signs (AVN and FAN) were associated with reduced retinal 
microvascular endothelial function, which underscores an important link between structure and function of retinal vessels.

Financial Disclosures: None

Funding Sources: Dr Al-Fiadh was supported by a co-funded Postgraduate Scholarship from the National Health and Medical Research Council (NHMRC) and National Heart Foundation of Australia (ID 567147). This work was supported in part by a Grant-in-Aid from the National Heart Foundation of Australia (G 08M 3781). 


\section{References}

1. Wang JJ, Liew G, Klein R, Rochtchina E, Knudtson MD, Klein BE, Wong TY, Burlutsky G, Mitchell P. Retinal vessel diameter and cardiovascular mortality: Pooled data analysis from two older populations. Eur Heart J. $2007 ; 28: 1984-1992$

2. Cheung N, Wang JJ, Klein R, Couper DJ, Sharrett AR, Wong TY. Diabetic retinopathy and the risk of coronary heart disease: The atherosclerosis risk in communities study. Diabetes Care. 2007;30:1742-1746

3. McGeechan K, Liew G, Macaskill P, Irwig L, Klein R, Klein BEK, Wang JJ, Mitchell P, Vingerling JR, Dejong PTVM, Witteman JCM, Breteler MMB, Shaw J, Zimmet P, Wong TY. Meta-analysis: Retinal vessel caliber and risk for coronary heart disease. Ann Intern Med. 2009;151:404-413

4. Wong TY, Kamineni A, Klein R, Sharrett AR, Klein BE, Siscovick DS, Cushman M, Duncan BB. Quantitative retinal venular caliber and risk of cardiovascular disease in older persons: The cardiovascular health study. Arch Intern Med. 2006;166:2388-2394

5. Wong TY, Klein R, Couper DJ, Cooper LS, Shahar E, Hubbard LD, Wofford MR, Sharrett AR. Retinal microvascular abnormalities and incident stroke: The atherosclerosis risk in communities study. Lancet. 2001;358:1134-1140

6. Wong TY, Klein R, Sharrett AR, Duncan BB, Couper DJ, Tielsch JM, Klein BE, Hubbard LD. Retinal arteriolar narrowing and risk of coronary heart disease in men and women. The atherosclerosis risk in communities study. JAMA. 2002;287:1153-1159 
7. Dorner GT, Garhofer G, Kiss B, Polska E, Polak K, Riva CE, Schmetterer L. Nitric oxide regulates retinal vascular tone in humans. Am J Physiol Heart Circ Physiol. 2003;285:H631-636

8. Nguyen TT, Kawasaki R, Kreis AJ, Wang JJ, Shaw J, Vilser W, Wong TY. Correlation of light-flicker-induced retinal vasodilation and retinal vascular caliber measurements in diabetes. Invest Ophthalmol Vis Sci. 2009;50:56095613

9. Seifertl BU, Vilser W. Retinal vessel analyzer (rva)--design and function. Biomed Tech (Berl). 2002;47 Suppl 1 Pt 2:678-681

10. Wong TY, Knudtson MD, Klein R, Klein BE, Meuer SM, Hubbard LD. Computer-assisted measurement of retinal vessel diameters in the beaver dam eye study: Methodology, correlation between eyes, and effect of refractive errors. Ophthalmology. 2004;111:1183-1190

11. Hubbard LD, Brothers RJ, King WN, Clegg LX, Klein R, Cooper LS, Sharrett AR, Davis MD, Cai J. Methods for evaluation of retinal microvascular abnormalities associated with hypertension/sclerosis in the atherosclerosis risk in communities study. Ophthalmology. 1999;106:2269-2280

12. Kawasaki R, Xie J, Cheung N, Lamoureux E, Klein R, Klein BE, Cotch MF, Sharrett AR, Shea S, Wong TY. Retinal microvascular signs and risk of stroke: The multi-ethnic study of atherosclerosis (mesa). Stroke.

$2012 ; 43: 3245-3251$

13. Landis JR, Koch GG. The measurement of observer agreement for categorical data. Biometrics. 1977;33:159-174 
14. Bland JM, Altman DG. A note on the use of the intraclass correlation coefficient in the evaluation of agreement between two methods of measurement. Computers in biology and medicine. 1990;20:337-340

15. Nguyen TT, Kreis AJ, Kawasaki R, Wang JJ, Seifert BU, Vilser W, Nagel E, Wong TY. Reproducibility of the retinal vascular response to flicker light in asians. Curr Eye Res. 2009;34:1082-1088

16. Chen HC, Patel V, Wiek J, Rassam SM, Kohner EM. Vessel diameter changes during the cardiac cycle. Eye (Lond). 1994;8 ( Pt 1):97-103

17. Sharrett AR, Hubbard LD, Cooper LS, Sorlie PD, Brothers RJ, Nieto FJ, Pinsky JL, Klein R. Retinal arteriolar diameters and elevated blood pressure: The atherosclerosis risk in communities study. Am J Epidemiol. $1999 ; 150: 263-270$

18. Lanigan LP, Clark CV, Hill DW. Retinal circulation responses to systemic autonomic nerve stimulation. Eye (Lond). 1988;2 ( Pt 4):412-417

19. Lam A, Bunya V, Piltz-Seymour JR. Visual field loss in patients with glaucoma who have asymmetric peripapillary focal arteriolar narrowing. Arch Ophthalmol. 2002;120:1494-1497

20. Wong TY, Wang JJ, Rochtchina E, Klein R, Mitchell P. Does refractive error influence the association of blood pressure and retinal vessel diameters? The blue mountains eye study. Am J Ophthalmol. 2004;137:1050-1055

21. Leung H, Wang JJ, Rochtchina E, Tan AG, Wong TY, Hubbard LD, Klein R, Mitchell P. Computer-assisted retinal vessel measurement in an older population: Correlation between right and left eyes. Clin Experiment Ophthalmol. 2003;31:326-330 
22. Wasan B, Cerutti A, Ford S, Marsh R. Vascular network changes in the retina with age and hypertension. J Hypertens. 1995;13:1724-1728

23. Puri R, Liew GY, Nicholls SJ, Nelson AJ, Leong DP, Carbone A, Copus B, Wong DT, Beltrame JF, Worthley SG, Worthley MI. Coronary beta2adrenoreceptors mediate endothelium-dependent vasoreactivity in humans: Novel insights from an in vivo intravascular ultrasound study. Eur Heart J. 2012;33:495-504

24. Howard G, Burke GL, Evans GW, Crouse JR, 3rd, Riley W, Arnett D, de Lacy R, Heiss G. Relations of intimal-medial thickness among sites within the carotid artery as evaluated by b-mode ultrasound. Aric investigators. Atherosclerosis risk in communities. Stroke. 1994;25:1581-1587

25. Thijssen DH, Dawson EA, Black MA, Hopman MT, Cable NT, Green DJ. Heterogeneity in conduit artery function in humans: Impact of arterial size. Am J Physiol Heart Circ Physiol. 2008;295:H1927-1934

26. Pyke KE, Tschakovsky ME. The relationship between shear stress and flowmediated dilatation: Implications for the assessment of endothelial function. $J$ Physiol. 2005;568:357-369

27. Nagel E, Vilser W, Lanzl I. Age, blood pressure, and vessel diameter as factors influencing the arterial retinal flicker response. Invest Ophthalmol Vis Sci. 2004;45:1486-1492

28. Polak K, Schmetterer L, Riva CE. Influence of flicker frequency on flickerinduced changes of retinal vessel diameter. Invest Ophthalmol Vis Sci. $2002 ; 43: 2721-2726$ 
29. Riva CE, Falsini B, Logean E. Flicker-evoked responses of human optic nerve head blood flow: Luminance versus chromatic modulation. Invest Ophthalmol Vis Sci. 2001;42:756-762

30. Klein R, Sharrett AR, Klein BE, Chambless LE, Cooper LS, Hubbard LD, Evans G. Are retinal arteriolar abnormalities related to atherosclerosis?: The atherosclerosis risk in communities study. Arterioscler Thromb Vasc Biol. 2000;20:1644-1650

31. Kreis AJ, Nguyen TT, Wang JJ, Rogers S, Al-Fiadh A, Freeman M, Wong TY, Farouque HM. Are retinal microvascular caliber changes associated with severity of coronary artery disease in symptomatic cardiac patients? Microcirculation. 2009;16:177-181

32. Mehlsen J, Jeppesen P, Erlandsen M, Poulsen PL, Bek T. Lack of effect of short-term treatment with amlodipine and lisinopril on retinal autoregulation in normotensive patients with type 1 diabetes and mild diabetic retinopathy. Acta Ophthalmol. 2011;89:764-768

33. Kern TS, Barber AJ. Retinal ganglion cells in diabetes. $J$ Physiol. 2008;586:4401-4408

34. Nguyen TT, Shaw JE, Robinson C, Kawasaki R, Wang JJ, Kreis AJ, Wong TY. Diabetic retinopathy is related to both endothelium-dependent and independent responses of skin microvascular flow. Diabetes Care. 2011;34:1389-1393

35. Flammer AJ, Anderson T, Celermajer DS, Creager MA, Deanfield J, Ganz P, Hamburg NM, Luscher TF, Shechter M, Taddei S, Vita JA, Lerman A. The assessment of endothelial function: From research into clinical practice. Circulation. 2012;126:753-767 
Figure 1. Dynamic Retinal Vascular Study. (A) Real time analysis of retinal venular (top - blue) and retinal arteriolar (bottom - red) diameter; $x$-axis is time in seconds and y-axis is vessel diameter in microns. The distance between the yellow bars represents the flickering light period. (B) Recording showing normal retinal vasodilator response to flicker light in a subject without CAD. (C) Markedly attenuated retinal vasodilator response in a subject with CAD. The dotted green lines represent the normal range of flicker light response. (D and E) Associated retinal photographs showing vessels of interest for dynamic vessel analysis.

Figure 2. Bland-Altman plots of the differences between the $1^{\text {st }}$ and $2^{\text {nd }}$ measurements acquired by the dynamic vessel analyzer against the average of 1 st and 2 nd measurement at baseline and at maximum dilatation after a flicker light stimulus. Dashed red line represents the mean difference and dashed green lines represent $95 \%$ limits of agreement (1.96 SD). (A) arteriolar baseline diameter; 0/13 outside the limit of agreement, mean difference -0.485 and $95 \%$ limit of agreement $(-3.582,2.612)$ (B) FI-RAD; $1 / 13(7.69 \%)$ outside the limit of agreement, mean difference -0.089 and 95\% limit of agreement $(-1.847,2.023)(\mathrm{C})$ venular baseline diameter; 0/13 outside the limit of agreement, mean difference 2.495 and $95 \%$ limit of agreement (-5.41, 10.40) and (D) FI-RVD; 0/13 outside the limit of agreement mean difference 0.358 and $95 \%$ limit of agreement $(-1.757,2.472)$.

Figure 3. (A) Scatterplots demonstrating a modest relationship between right and left eyes. (B) Scatterplots demonstrating an inverse relationship between baseline retinal arteriolar diameter and FI-RAD. 
Figure 4. (A) Flicker light induced retinal arteriolar dilatation (FI-RAD) is attenuated in patients with arteriovenous nicking $(\mathrm{AVN})$ compared to non-AVN $(1.43 \pm 0.2 \% v s$ $2.28 \pm 0.17 \%$ ); and (B) focal arteriolar narrowing (FAN) compared to non-FAN $(1.20 \pm 0.19 \%$ vs $2.21 \pm 0.16 \%)$. Data are presented as mean \pm SEM and independent samples $t$-test was used to compare means. 


\begin{tabular}{|c|c|}
\hline Variable $(n=258)$ & Mean \pm SD or number $(\%)$ \\
\hline Age (years) & $58.2 \pm 11.0$ \\
\hline Gender (male) & $175(67.8)$ \\
\hline Hypertension & $187(72.5)$ \\
\hline Dyslipidaemia & $200(77.5)$ \\
\hline Current smoking & $72(27.9)$ \\
\hline Diabetes mellitus & $88(35.7)$ \\
\hline Body mass index $\left(\mathrm{kg} / \mathrm{m}^{2}\right)$ & $31.8 \pm 6.9$ \\
\hline Non-coronary artery disease & $119(46.1)$ \\
\hline Coronary artery disease & $139(53.9)$ \\
\hline Systolic blood pressure (mmHg) & $137 \pm 19$ \\
\hline Diastolic blood pressure $(\mathrm{mmHg})$ & $76 \pm 11$ \\
\hline Diabetes medication & $60(23.3)$ \\
\hline Statin therapy & $157(60.9)$ \\
\hline RAS blocker medication & $156(60.5)$ \\
\hline Beta blocker & $88(34.1)$ \\
\hline Calcium channel blocker & $55(21.3)$ \\
\hline Serum creatinine $(\mu \mathrm{mol} / \mathrm{L})$ & $80.0 \pm 31.8$ \\
\hline Glucose (mmol/L) & $6.4 \pm 2.4$ \\
\hline HbA1c $(\%)$ & $7.4 \pm 1.7$ \\
\hline Cholesterol (mmol/L) & $4.5 \pm 1.3$ \\
\hline Triglyceride (mmol/L) & $1.5 \pm 1.17$ \\
\hline $\mathrm{HDL}(\mathrm{mmol} / \mathrm{L})$ & $1.1 \pm 0.4$ \\
\hline
\end{tabular}

Table 1. Characteristics of the study cohort. Data expressed as either mean \pm SD or number (\%). RAS = renin-angiotensin system. 


\begin{tabular}{|l|c|c|c|c|}
\hline $\begin{array}{l}\text { Retinal vascular } \\
\text { measures }(\mathbf{n}=\mathbf{2 5 8})\end{array}$ & Right eye & Left eye & Coefficient & $\boldsymbol{p}$ \\
\hline BL-RAD $(\mu \mathrm{m})$ & $120.33 \pm 15.20$ & $118.74 \pm 13.45$ & $r=0.44$ & $<0.001$ \\
\hline BL-RVD $(\mu \mathrm{m})$ & $148.72 \pm 19.70$ & $150.68 \pm 21.73$ & $r=0.35$ & $<0.001$ \\
\hline FI-RAD $(\%)$ & $1.91 \pm 2.12$ & $2.00 \pm 2.06$ & $r=0.58$ & $<0.001$ \\
\hline FI-RVD $(\%)$ & $4.02 \pm 2.30$ & $3.59 \pm 1.91$ & $r=0.59$ & $<0.001$ \\
\hline CRAE $(\mu \mathrm{m})$ & $138.59 \pm 13.33$ & $138.03 \pm 12.72$ & $r=0.76$ & $<0.001$ \\
\hline CRVE $(\mu \mathrm{m})$ & $215.39 \pm 19.77$ & $215.48 \pm 20.61$ & $r=0.82$ & $<0.001$ \\
\hline AVN, $\mathrm{n}(\%)$ & $93(36.0)$ & $80(31.0)$ & $\kappa=0.18$ & 0.004 \\
\hline FAN, $\mathrm{n}(\%)$ & $55(21.3)$ & $49(19.0)$ & $\kappa=0.42$ & $<0.001$ \\
\hline
\end{tabular}

Table 2. Associations of retinal parameters between right and left eyes. Data presented as mean \pm standard deviation or number [(n (\%)]. Pearson's correlation $(r)$ for retinal vessel calibre and flicker-light induced vascular dilatation; Kappa statistic correlation $(\kappa)$ for retinal structural changes between right and left eyes; BL-RAD = baseline retinal arteriolar diameter $(\mu \mathrm{m})$; BL-RVD = baseline retinal venular diameter $(\mu \mathrm{m}) ;$ FI-RAD $=$ flicker-light induced retinal arteriolar dilatation $(\%)$; FI-RVD $=$ flicker-light induced retinal venular dilatation $(\%)$; CRAE $=$ central retinal artery equivalent $(\mu \mathrm{m}) ; \mathrm{CRVE}=$ central retinal vein equivalent $(\mu \mathrm{m}) ; \mathrm{AVN}=$ arteriovenous nicking; FAN = focal arteriolar narrowing. 
Figure 1.
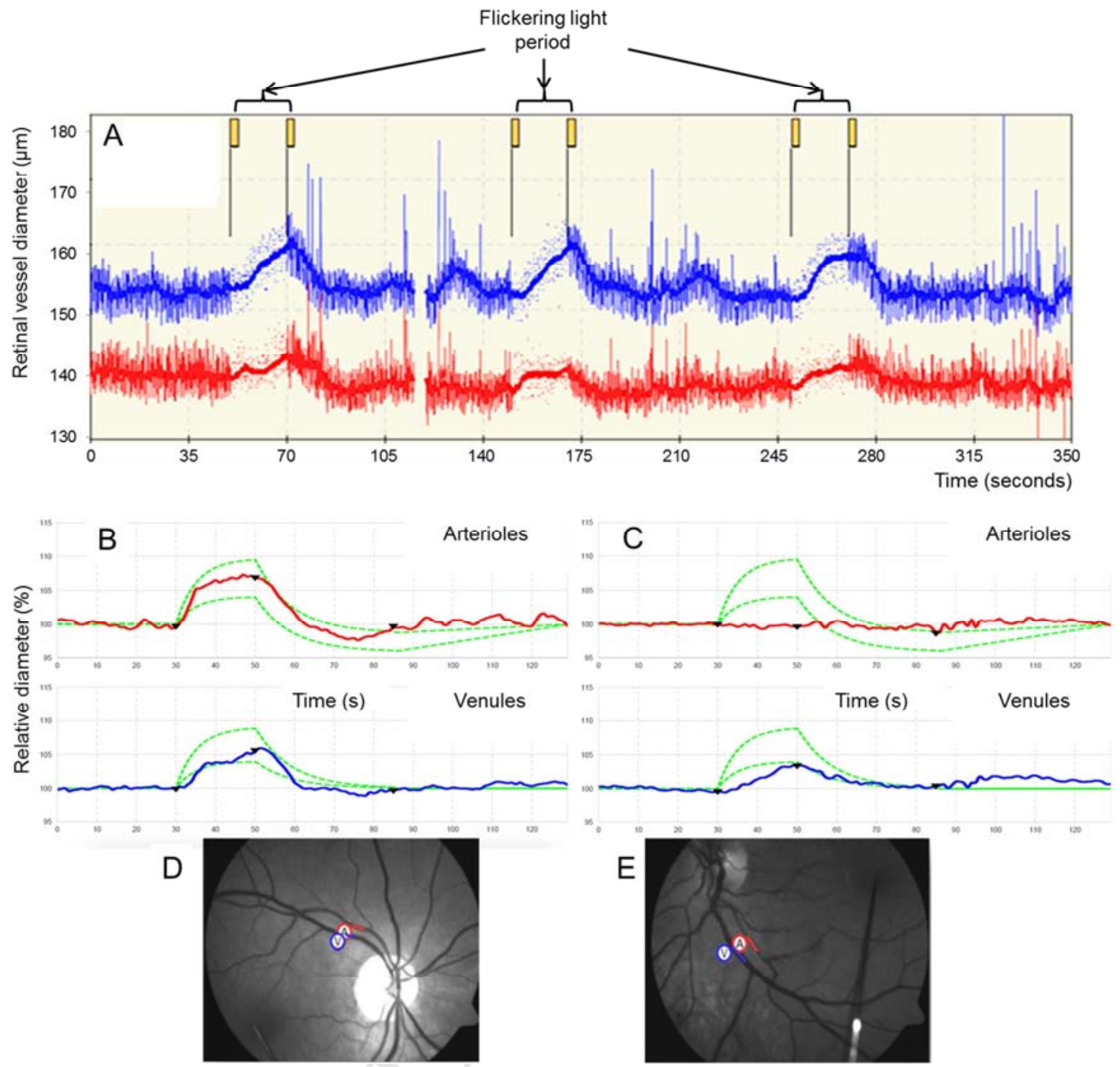
Figure 2.
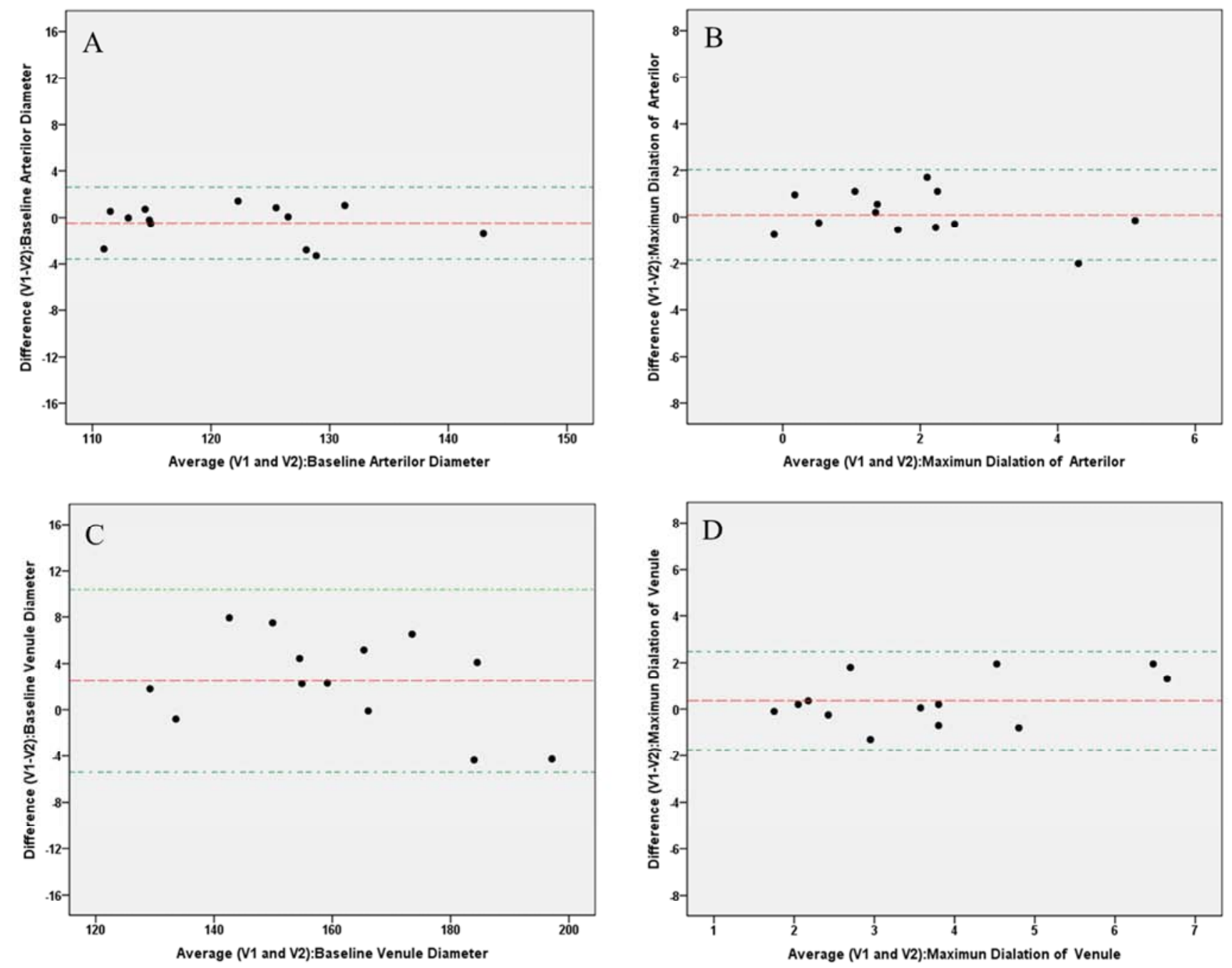
Figure 3.
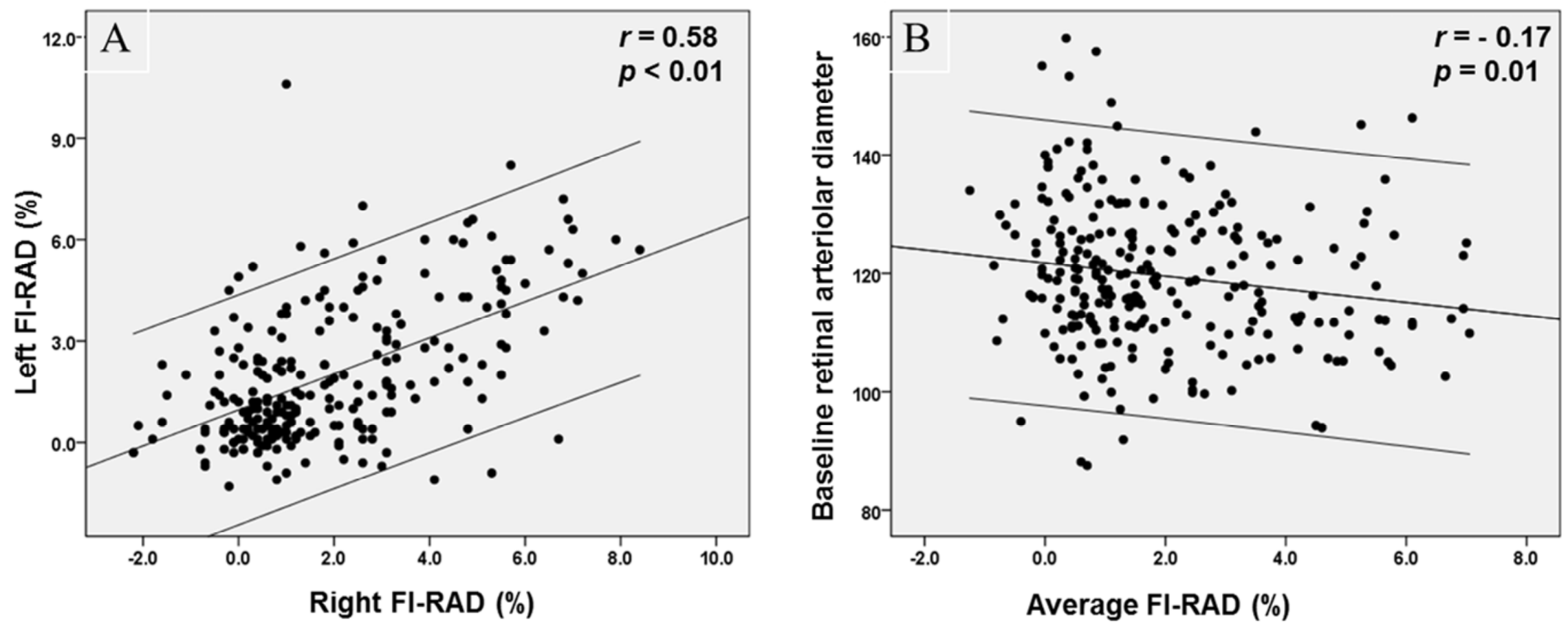
Figure 4.
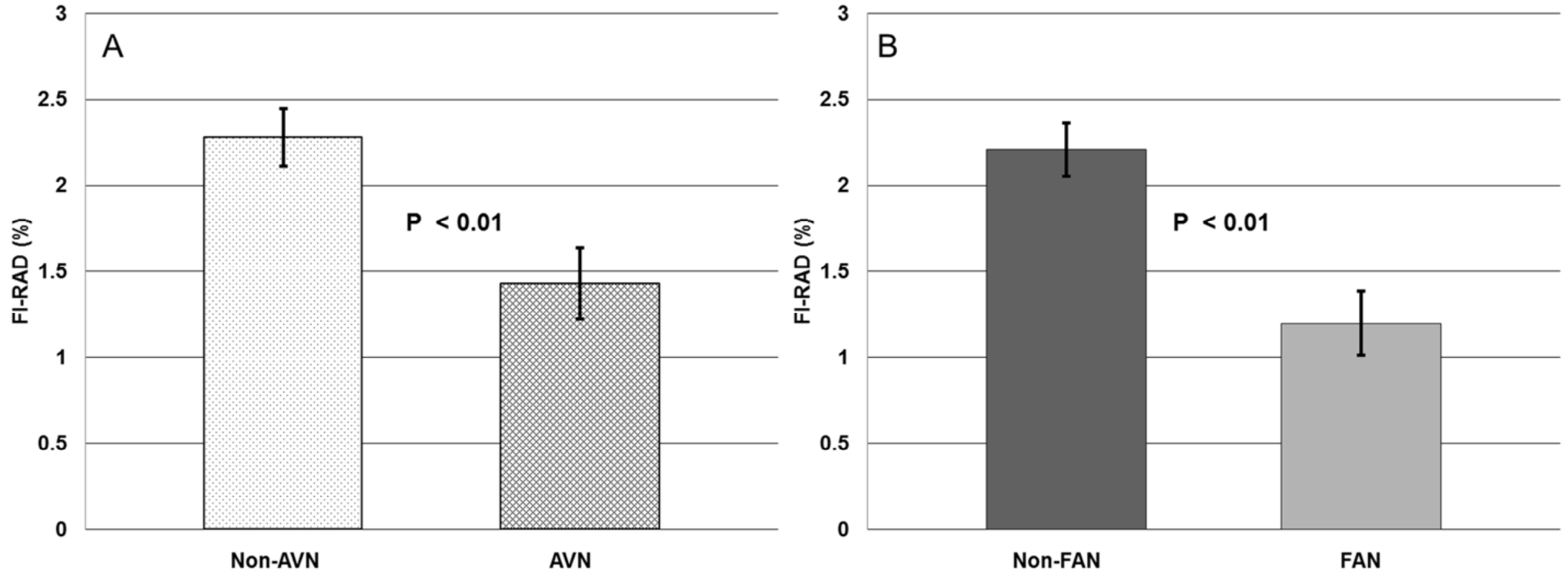


\section{Highlights}

- Retinal microvascular endothelial function was assessed by Dynamic Vessel Analyzer (DVA)

- The DVA measurements are reproducible in patients at risk of coronary artery disease

- Right and left eyes respond in a similar manner

- Smaller retinal vessels were associated with an increased flicker response

- Retinal microvascular structure changes was associated with reduced flicker response 


\section{University Library}

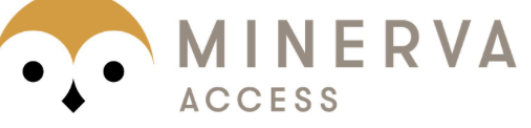

A gateway to Melbourne's research publications

Minerva Access is the Institutional Repository of The University of Melbourne

Author/s:

Al-Fiadh, AH;Farouque, O;Kawasaki, R;Nguyen, TT;Uddin, N;Freeman, M;Patel, SK;Burrell, LM;Wong, TY

Title:

Retinal microvascular structure and function in patients with risk factors of atherosclerosis and coronary artery disease

Date:

2014-04-01

Citation:

Al-Fiadh, A. H., Farouque, O., Kawasaki, R., Nguyen, T. T., Uddin, N., Freeman, M., Patel, S. K., Burrell, L. M. \& Wong, T. Y. (2014). Retinal microvascular structure and function in patients with risk factors of atherosclerosis and coronary artery disease. ATHEROSCLEROSIS, 233 (2), pp.478-484. https://doi.org/10.1016/ j.atherosclerosis.2013.12.044.

Persistent Link:

http://hdl.handle.net/11343/43930 